\title{
Interacting Quantum Fields Around a Black Hole
}

\author{
S. W. Hawking \\ University of Cambridge, D.A.M.T.P. Silver Street, Cambridge, England
}

\begin{abstract}
If one studies interacting fields on a black hole background using ordinary Feynman diagrams, one is faced with a problem of what to do with lines that cross the horizon. To avoid this difficulty a formulation is developed which can be expressed graphically in terms of a new class of diagram with external lines only at infinity. This formalism is applied to study the question of whether spontaneously broken symmetry would be restored near the black hole. It is also used to show that a black hole can emit more particles than antiparticles even in theories where the particle number is locally conserved by a global $U(1)$ symmetry.
\end{abstract}

\section{Introduction}

The theory of non-interacting quantum fields in the vicinity of a black hole is now well understood in the semi-classical approximation in which the gravitional field of the black hole can be regarded as a fixed stationary background. One finds that the black hole creates and emits particles as if it were a hot body [1] with a temperature proportional to the surface gravity of the black hole [2]. (In the case of an uncharged non-rotating black hole,

$$
T=(8 \pi M)^{-1}
$$

in units in which $G=c=\hbar=k=1$, where $M$ is the mass of the black hole.) One can understand this emission heuristically as follows: The Uncertainty Principle implies that "empty" spacetime is filled with closed loops of particles. One can visualise one of these as corresponding to a particle-antiparticle pair which appear together, move apart and them come together again and annihilate each other. They cannot continue to exist as "real" particles because in that case they would both have positive energy which would violate the conservation of energy. However when a black hole is present, one of the pair can tunnel throught the horizon into the interior where there are particle states which have negative energy with respect to infinity. This allows the other particle to escape to infinity as a real particle 
with positive energy. The flux of negative energy across the horizon reduces the mass of the black hole and causes the horizon to shrink. However the rate of change of the metric $\sim M^{-3}$ is very slow compared to the typical frequency of the radiation $\sim M^{-1}$ as long as $M \gg 1$, i.e. the Planck mass $\sim 10^{-5} \mathrm{~g}$. It is therefore a good approximation to neglect the time dependence of the metric and to calculate the emission from a sequence of stationary metrics.

A consequence of the pair creation picture is that the radiation at infinity appears to an observer there to be described by a density matrix rather than by a pure state. This is because such an observer cannot even in principle observe the particles that fell into the black hole. Thus he has to reduce the pure quantum state describing the particle-antiparticle pair by summing over all possible states for the unobserved particle. In fact it turns out that the density matrix describing the emitted radiation is completely thermal [3-5], i.e. the black hole emits with equal probability every configuration of particles with a given energy though not every configuration escapes to infinity with equal probability because there is a potential barrier around the black hole which depends on spin, angular momentum and energy of the individual particles and which may reflect some of them back into the black hole. The black hole can be in thermal equilibrium with black body radiation at the same temperature though the equilibrium is not stable unless the total energy is fixed by placing the whole system in an insulated box of a certain size [6]. The underlying reason for the thermal character of the radiation from black holes is that the black hole metrics can be analytically continued to "Euclidean," i.e. positive definite, metrics which are periodic in the imaginary time coordinate $\tau=-$ it with period $\beta[7-10]$. This means that any fields which are analytic in the real Lorentzian black hole metric are periodic in the imaginary time coordinate and so behave as if they were at finite temperature $T=\beta^{-1}$.

One would like to know how these results for free fields on a black hole background are modified by the presence of interactions. Strictly speaking, one ought to include the interactions with the gravitational field itself. That would require a full quantum theory of gravity which does not yet exist. However for black holes significantly bigger than the Planck mass, the interactions between the various matter fields will be much greater than their interactions with the gravitional field and so, as before, it will be a very good approximation to consider only the interactions between the matter fields on a fixed black hole background. Interacting field theories in curved spacetime backgrounds seem to be renormalizable provided they are renormalizable in flat spacetime (see [11] for a review). It would therefore appear straightforward to calculate the effeect of interactions on black hole emission: one would just write down a string of Feynman diagrams where the lines correspond to propagators that were solutions of the free field wave equation in the curved spacetime background. The trouble with this is that one would have to include diagrams with lines that crossed the horizon and which represented particles entering or leaving the black hole. One would not know what factor to put at the end of these lines. One's first guess might be to put the thermal factor corresponding to free field particle states. However this would not be correct because the interactions are likely to be important near the horizon so that the true spectrum could be very different from the free particle thermal spectrum. Furthermore, such a procedure would lead to unphysical divergences near the 
horizon because there would appear to be thermal states of very high angular momentum which would be emitted from the black hole and almost totally reflected back into it.

I shall therefore adopt a different procedure which can be formulated in terms of a new kind of perturbation diagram which has external lines only at past and future infinity and which does not have any lines crossing the horizon. Ordinary Feynman diagrams give the amplitudes for the various possible final pure quantum states. However the radiation from a black hole is described by a density matrix rather than a pure quantum state. This new class of diagrams directly measures certain moments of the density matrix of the outgoing radiation. These diagrams can be regarded as combinations of Feynman diagrams for the amplitude for a certain number of particles to escape to infinity and to fall into the black hole multiplied by the complex conjugate diagrams and summed over the unobserved particle states. However this summation over internal states is automatic: one does not have to specify the behaviour on the horizon except to assume the fields are regular there.

One first defines Green's functions for the interacting fields on the Euclidean section of the black hole metric by standard perturbation methods using the free field propagators on the curved space background. The renormalization of these Green's functions is essentially similar to that of Green's functions in flat Euclidean space with the possible addition of a $\phi^{2} R$ counterterm (such a counterterm will be absent on black hole backgrounds because $R \equiv 0$ ). We do not have a closed form for the free field propagators but the renormalization can be performed numerically at least to one loop by zeta function regularization [12]. One then analytically continues these Green's functions from the Euclidean regime of imaginary $t$ where they are defined to the Lorentzian regime of real $t$. One interprets the Green's functions not as matrix elements of time ordered products of field operators between an "in" and "out" vacua but as expectation values in a mixed state described by a density matrix. The fact that the Euclidean metric is periodic in the imaginary time coordinate means that these Green's functions will describe a situation in which there is a black hole in equilibrium with thermal radiation. The radiation will be strictly thermal in the presence of interactions [8]. However the outgoing radiation at infinity will not appear to have an exactly thermal spectrum when analysed in terms of free particle states because these are not energy eigenfunctions of the full, interacting Hamiltonian. To determine the spectrum in terms of free particle states one uses the interacting Green's functions analytically continued from the Euclidean section to propagate from a point at future infinity slightly above the real $t$ axis to a similar point slightly below. One obtains a series of perturbation diagrams with external lines going to points at future infinity above and below the real $t$-axis. If the Green's functions had been strictly causal (as in flat space at zero temperature), these diagrams would have been zero. However the periodicity in imaginary time of the background metric means that the Green's functions will have singularities periodically distributed up the imaginary time axis and these will give a non-zero contribution.

The above procedure allows one to determine the spectrum of outgoing radiation from a black hole in equilibrium with thermal radiation. However this is not a situation that one is likely to encounter; what one would like to calculate is 
the emission from a black hole radiating into empty space with no incoming radiation. One therefore has to substract out the effects of the incoming radiation from the equilibrium spectrum. One does this by replacing each line in the equilibrium diagrams by a pair of propagators from points at past infinity above and below the real $t$-axis. On therfore obtains a series of diagrams with external lines at past and future infinity. The sum of all such diagrams (with appropriate signs) and the equilibrium diagrams gives the spectrum or higher moments of the density matrix of the outgoing radiation for a black hole radiating into empty space.

Using this formalism one could calculate the QED corrections to the emission of photons, electrons and positrons. Page [13] has shown that there are corrections of order $e^{2}$ caused by the fluctuating charge on the black hole. There will also be other corrections of the same order from one loop effects. These could be calculated by using numerical zeta function techniques. However one would expect the effects to be small. Of more interest are situations in which interactions can produce qualitatively new effects. Two examples are discussed in Sect. 6 and 7. The first is spontaneous symmetry breaking. One would expect that symmetry might be restored near the black hole if the temperature were above a certain critical value. On the other hand one would not expect the presence of the black hole to effect the symmetry breaking a long way away. This might cause an explosive outburst when a black hole got down to about $10^{11} \mathrm{~g}$, at which point the temperature would be of the order of the electroweak unification energy of about $100 \mathrm{GeV}$. The other possible effect that is discussed is the emission of different numbers of particles and antiparticles. Such particle-anti-particle assymetry can occur in Grand Unified Theories which do not conserve baryon number locally. On the other hand, it has been shown [14] that there cannot be any net asymmetry between the emission of particles and antiparticles in theories that have local conservation of particle number and in which the particles interact only with the background metric, even if these interactions violate $\mathrm{CP}$. However this result does not apply to fields which interact with themselves and each other. An example is given of an interacting field theory which conserves particle number locally but which would give different rates of emission for particles and antiparticles. It is probable that this effect is not of much practical significance for black holes substantially bigger than the Planck mass but its importance lies in the fact that it demonstrates that gravitational interactions can produce baryon asymmetry without recourse to Grand Unified Theories. One could think of the initial singularity of the universe as being like a whole collection of Planck mass black holes. Presumably it could therefore give rise to a universe with a net baryon number.

The plan of the paper is as follows:

In Sect. 2 Green's functions for free and interacting fields are defined on the Euclidean section of the black hole metric. Their analytic structure and Fourier transforms with respect to real time are discussed. In Sect. 3 the asymptotic values of the Euclidean Green's functions at large radii are interpreted in terms of annihilation and creation operators for ingoing and outgoing particles in thermal equilibrium. The number operator for outgoing particles is expressed in terms of diagrams with two outgoing external lines. In Sect. 4 the effect of the incoming 
radiation is substracted out by replacing the Euclidean free field propagators by the Unruh propagator. This can be expressed in terms of the Euclidean propagator and leads to diagrams with incoming and outgoing external lines. As an application of this formalism it is shown in Sect. 5 how to calculate the expectation value of $|\phi|^{2}$ and $T^{\mu \nu}$. Section 6 deals with the possible restoration of spontaneously broken symmetry near the black hole and Sect. 7 discusses the possible asymetric emission of particles and antiparticles.

\section{The Euclidean Green's Functions}

The Lorentzian (signature -+++ ) Schwarzschild metric is normally given in the form

$$
d s^{2}=-\left(1-2 M r^{-1}\right) d t^{2}+\left(1-2 M r^{-1}\right)^{-1} d r^{2}+r^{2}\left(d \theta^{2}+\sin ^{2} \theta d \phi^{2}\right) .
$$

If one puts $\tau=+\mathrm{it}$, this gives a positive definite (Euclidean) metric for $r>2 M$. There is an apparent singularity at $r=2 M$ but this can be removed by introducing a new radial coordinate

$$
x=4 M\left(1-2 M r^{-1}\right)^{1 / 2}
$$

Then the metric becomes

$$
d s^{2}=x^{2}\left(\frac{d \tau}{4 M}\right)^{2}+\left(\frac{r}{2 M}\right)^{4} d x^{2}+r^{2} d \Omega^{2} .
$$

The apparent singularity at $r=2 M, x=0$ is thus just like the origin of polar coordinates and will be removed if $\tau / 4 M$ is regarted as an angular coordinate and is identified with period $2 \pi$, i.e. $\tau$ is identified with period $8 \pi M$. One therefore obtains a complete singularity free positive definite (Euclidean) metric which is periodic in the imaginary time coordinate $\tau$. Integer spin quantum fields on this background are thus automatically periodic in imaginary time and so contribute to the canonical ensemble at a temperature $T=(8 \pi M)^{-1}$.

In the case of half-integer spins, a rotation of $2 \pi$ in the angular coordinate $\tau / 4 M$ about the "axis" $x=0$ will change the sign of the field. Thus fermion fields on the Euclidean section are antiperiodic in imaginary time which is again how they should be for the canonical ensemble.

In a similar manner one can find a complete, singularity free, Euclidean section of the Kerr metrics though in this case the angular momentum $J$ has to be taken to be imaginary. Fields on such a background will contribute to the grand canonical ensemble in which, in addition to a temperature there is a chemical potential (the angular velocity) for angular momentum. One then analytically continues the angular momentum $J$ of the black hole to real values.

The Euclidean Green's functions for the free fields are defined to be the unique solutions of the relevant inhomogeneous wave equations that are regular on the Euclidean section and die away at large values of $r$. For example, the Green's function of a scalar field of mass $m$ obeys the equation

$$
\left(-\square_{x}+m^{2}\right) G_{0}(x, y)=\delta(x, y) \text {. }
$$


It can be represented as

$$
G_{0}(x, y)=\sum_{n} \frac{\phi_{n}^{*}(x) \phi_{n}(y)}{\lambda_{n}}
$$

where $\phi_{n}$ and $\lambda_{n}$ are the eigenfunctions and eigenvalues of the elliptic operator $-\square+m^{2}$ on the Euclidean section

$$
\left(-\square+m^{2}\right) \phi_{n}=\lambda_{n} \phi_{n} .
$$

The eigenfunctions are normalized by

$$
\int \phi_{n}^{*}(x) \phi_{m}(x) \sqrt{g} d^{4} x=\delta_{m n} .
$$

Strictly speaking, the summation in (2.5) ought to be replaced by an integral and the Kronecker delta in (2.7) ought to be replaced by a $\delta$-function because the spectrum of the wave operator will be continuous since the Euclidean section is unbounded. However, for numerical calculations it is convenient to introduce a boundary at a large value of $r$ and to impose Dirichlet or Neumann boundary conditions there. Near to the boundary this will introduce unphysical divergences of the energy momentum tensor etc. [15-17]. However, if the boundary is at large radius it will not have much effect on the Green's functions near the black hole which is where the interactions will be concentrated. In the case of the Schwarzschild metric the eigenfunctions can be written in the form

$$
\phi_{n}=\frac{1}{r} \exp \left(\frac{i p \tau}{4 M}\right) Y_{\ell m}(\theta, \phi) R_{p \ell n}(r),
$$

where the radial function obeys the equation

$$
\left(1-2 M r^{-1}\right) \frac{d}{d r}\left[\left(1-2 M r^{-1}\right) \frac{d R}{d r}\right]-V(r) R=-\lambda_{n}\left(1-2 M r^{-1}\right) R,
$$

where

$$
V(r)=\left(\frac{p}{4 M}\right)^{2}+\left(1-2 M r^{-1}\right)\left[\ell(\ell+1) r^{-2}+2 M r^{-3}+m^{2}\right] .
$$

They are regular at $r=2 M$ and are zero at the boundary.

On the Euclidean section $G_{0}$ will be singular only when the two points are coincident. However if one continues $G_{0}(2,1)$ to the complex $t_{2}-t_{1}$ plane for fixed $r_{1}, \theta_{1}, \phi_{1}$ and $r_{2}, \theta_{2}, \phi_{2}$ there will be a singularity whenever the points 1 and 2 can be joined by a null geodesic (Fig. 1). These singularities will be periodically repeated in the imaginary $t$ direction because the Euclidean section is periodic in the imaginary time coordinate.

One can Fourier analyse $G_{0}(2,1)$ in terms of $t_{2}-t_{1}$. If $\operatorname{Im}\left(t_{2}-t_{1}\right)>0$, the contour lies above the real axis in figure 1 and

$$
\begin{aligned}
G_{0}(2,1)= & \frac{1}{4 \pi r^{2}} \sum_{\ell, m} \int_{-\infty}^{\infty} d \omega e^{-i \omega\left(t_{1}-t_{2}\right)} \cdot\left(1-e^{-\beta \omega}\right)^{-1} \cdot \operatorname{sgn}(\omega) \\
& \cdot Y_{\ell m}^{*}\left(\theta_{2}, \phi_{2}\right) Y_{\ell m}\left(\theta_{1}, \phi_{1}\right) \\
& \cdot\left\{\vec{R}_{ \pm}^{*}\left(r_{2}\right) \vec{R}_{ \pm}\left(r_{1}\right)+\overleftarrow{R}_{ \pm}^{*}\left(r_{2}\right) \overleftarrow{R}_{ \pm}\left(r_{1}\right)\right\}
\end{aligned}
$$




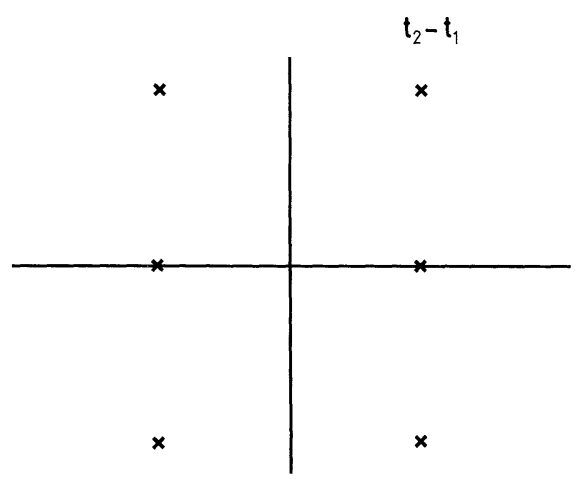

Fig. 1. The Euclidean propagator $G_{0}(2,1)$ contains singularities that are periodically repeated in the complex $t_{2}-t_{1}$ plane

If $\operatorname{Im}\left(t_{2}-t_{1}\right)<0$, the positions of 1 and 2 on the right of 2.10 are reversed. Note that the Green's function contains both positive and negative frequencies whereas, in flat space at zero temperature it would contain only positive frequencies. This is because there is a contribution from the repeated singularities in the upper half $t_{2}-t_{1}$ plane. The negative frequency component in less than the positive frequency component by the Boltzman factor $e^{-\beta \omega}$. The radial function $\vec{R}_{-}$is the solution of the radial equation

$$
\frac{d^{2} R}{d r^{\prime 2}}-V(r) R=0
$$

where

$$
r^{\prime}=r+2 M \log \left(1-2 M r^{-1}\right),
$$

and where

$$
V(r)=-\omega^{2}+\left(1-2 M r^{-1}\right)\left[\ell(\ell+1) r^{-2}+2 M r^{-3}+m^{2}\right]
$$

with the boundary conditions that

$$
\begin{aligned}
\vec{R}_{-} & =e^{+i \omega r^{\prime}}+\vec{A}(\omega) e^{-i \omega r^{\prime}} \quad \text { as } \quad r^{\prime} \rightarrow-\infty \\
& =\left(\frac{\omega}{k}\right)^{1 / 2} \vec{B}(\omega) e^{+i k r^{\prime}} \quad \text { as } \quad r^{\prime} \rightarrow \infty,
\end{aligned}
$$

where $k=\left(\omega^{2}-m^{2}\right)^{1 / 2} \operatorname{sgn}(\omega)$. In other words, $\vec{R}_{-}$represents a wave of frequency $\omega$ in the Lorentzian Schwarzschild metric that emerges from the past horizon with unit amplitude and which is partially reflected back into the future horizon (with probability $|\vec{A}(\omega)|^{2}$ ) and partly transmitted to infinity as a purely outgoing wave (with probability $|\vec{B}(\omega)|^{2}=1-|\vec{A}(\omega)|^{2}$ ) (Fig. 2). $\vec{B}(\omega)$ is defined to be zero for $|\omega|<m$.

Similarly, $\overleftarrow{R}_{-}$is the solution of the radial equation that represents an incoming wave of amplitude $(\omega / k)^{1 / 2}$ from past infinity which is partially reflected to an outgoing wave at future infinity (with probability $|\overleftarrow{A}(\omega)|^{2}=|\vec{A}(\omega)|^{2}$ ) and partly transmitted to a purely ingoing wave on the future horizon (with probability $|\overleftarrow{B}(\omega)|^{2}=|\vec{B}(\omega)|^{2}=1-|\vec{A}(\omega)|^{2}$ ) (Fig. 2). $\overleftarrow{R}_{-}$is defined to be zero for $|\omega|<m$. The 

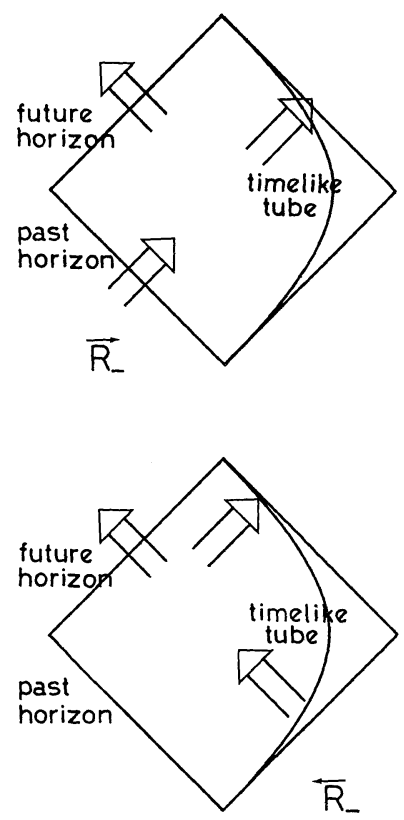

Fig. 2. The solution $\vec{R}_{\text {_ }}$ represents a wave that emerges from the past horizon and is partially reflected back into the future horizon. $\bar{R}_{-}$represents a wave that comes in from infinity and is partially reflected back to infinity

alternative basis $\vec{R}_{+}$and $\overleftarrow{R}_{+}$are defined in a similar manner with past and future interchanged.

The Euclidean Green's functions of an interacting field theory can now be constructed in the usual diagrammatic manner where the lines correspond to the Euclidean Green's function of the free field and the vertices represent terms in the interaction Lagrangian. One then integrates the positions of the vertices over the Euclidean section of the metric. As in flat space this will give rise to divergences which have to be rendered finite by some technique such as Pauli-Villars, dimensional or zeta-function regularization. The undefined quantities introduced by the regularization are then absorbed into a redefinition of the parameters such as masses or coupling constants. This renormalization is difficult to perform in practice because one does not have a closed from for the radial functions or a composition law for them. However for certain one loop diagrams, one can apply numerical zeta function regularization [12]. The one loop renormalization of the effective action for a scalar field will be considered in Sect. 6 .

\section{The Interpretation of the Green's Functions}

By construction, the interacting Green's functions are periodic or antiperiodic in the imaginary time coordinate. They are therefore best interpreted as the 
expectation values of products of the field operators, not in some pure quantum state, but in a mixed state with the density matrix

$$
\varrho=e^{-\beta H},
$$

where $H$ is the full, interacting, Hamiltonian. Thus, for example, the interacting two point function is

$$
G(x, y)=\frac{\operatorname{Tr}(\bar{\phi}(x) \phi(y) \varrho)}{\operatorname{Tr} \varrho} .
$$

The usual time ordering operator has been omitted deliberately. I shall return to operator ordering below.

I shall make the usual interaction picture assumption that the interactions can be neglected near infinity. This means that the field operators $\phi(x)$ and $\bar{\phi}(x)$ will obey the free field equations in the asymptotic region. They can therefore be represented in terms of annihilation and creation operators for incoming and outgoing waves at infinity

$$
\begin{aligned}
\phi(x)= & \sum_{\ell, m} \int_{m}^{\infty} d \omega\left(f_{\text {out }}(\ell, m ; \omega) a_{\text {out }}(\ell, m ; \omega)\right. \\
& +f_{\text {in }}(\ell, m ; \omega) a_{\text {in }}(\ell, m ; \omega) \\
& +f_{\text {out }}^{*}(\ell, m ; \omega) b_{\text {out }}^{+}(\ell, m ; \omega) \\
& \left.f_{\text {in }}^{*}(\ell, m ; \omega) b_{\text {in }}^{+}(\ell, m ; \omega)\right) \\
\bar{\phi}(x)= & \sum_{\ell, m} \int_{m}^{\infty} d \omega\left(f_{\text {out }}^{*}(\ell, m ; \omega) a_{\text {out }}^{+}(\ell, m ; \omega)\right. \\
& +f_{\text {in }}^{*}(\ell, m ; \omega) a_{\text {in }}^{+}(\ell, m ; \omega) \\
& +f_{\text {out }}(\ell, m ; \omega) b_{\text {out }}(\ell, m ; \omega) \\
& \left.+f_{\text {in }}(\ell, m ; \omega) b_{\text {in }}(\ell, m ; \omega)\right),
\end{aligned}
$$

where

$$
f_{\text {out }}(\ell, m ; \omega)=\frac{Y_{\ell m}}{(4 \pi k)^{1 / 2} r} \exp -i\left(\omega t-k r^{\prime}\right)
$$

and

$$
f_{\mathrm{in}}(\ell, m ; \omega)=\frac{Y_{\ell m}}{(4 \pi k)^{1 / 2} r} \exp -i\left(\omega t+k r^{\prime}\right)
$$

The operators $a_{\mathrm{out}}(\ell, m ; \omega)$ and $a_{\mathrm{out}}^{+}(\ell, m ; \omega)$ are the annihilation and creation for an outgoing particle of frequency $\omega$ with angular quantum numbers $\ell$ and $m(I$ shall normally omit the indices $\ell, m)$. Similarly $a_{\mathrm{in}}(\ell, m ; \omega)$ and $a_{\mathrm{in}}^{+}(\ell, m ; \omega)$ are the operators for an ingoing particle and the $b$ 's are the corresponding operators for antiparticles.

The "out" operators all commute with each other apart from

$$
\begin{aligned}
& {\left[a_{\text {out }}(\ell, m ; \omega), a_{\text {out }}^{+}\left(\ell^{\prime}, m^{\prime} ; \omega^{\prime}\right)\right]=\delta_{\ell \ell^{\prime}} \delta_{m m^{\prime}} \delta\left(\omega-\omega^{\prime}\right)} \\
& {\left[b_{\text {out }}(\ell, m ; \omega), b_{\text {out }}^{+}\left(\ell^{\prime}, m^{\prime} ; \omega^{\prime}\right)\right]=\delta_{\ell \ell^{\prime}} \delta_{m m^{\prime}} \delta\left(\omega-\omega^{\prime}\right) .}
\end{aligned}
$$


The "in" operators obey similar commutation relations among themselves and the $a_{\text {in }}$ and $b_{\text {in }}$ operators commute with the $a_{\text {out }}$ and $b_{\text {out }}$ operators but not, in general, with the $a_{\text {out }}^{+}$and $b_{\text {out }}^{+}$.

One can use the orthonormal properties of the basis functions under the normal scalar product to express the annihilation and creation operators in terms of the field operators. Thus, for example,

$$
a_{\text {out }}(\omega)=-i \int f_{\text {out }}^{*}(\omega ; x) \overleftrightarrow{V}_{\mu} \phi(x) d \Sigma^{\mu}(x),
$$

where the integral is taken over a timelike surface at large radius. By operating on the thermal density matrix $\varrho$ with various annihilation and creation operators one can measure different moments of the density matrix in terms of a basis of free particle states. The density matrix will not appear thermal in terms of this basis because the free particle states are not eigenstates of the full, interacting Hamiltonian. For example, the expectation value of the number of outgoing particles in the mode $f_{\text {out }}(\omega)$ will be

$$
\begin{aligned}
\left\langle n_{\text {out }}(\omega)\right\rangle= & \frac{\operatorname{Tr}\left(a_{\text {out }}^{+}(\omega) a_{\text {out }}(\omega) \varrho\right)}{\operatorname{Tr} \varrho} \\
= & \iint d \Sigma^{\mu}(x) d \Sigma^{v}(y) f_{\text {out }}(\omega ; x) f_{\text {out }}^{*}(\omega ; y) \\
& \cdot \overleftrightarrow{\nabla}_{\mu} \overleftrightarrow{\nabla}_{v}\left\{\frac{\operatorname{Tr}(\bar{\phi}(x) \phi(y) \varrho}{\operatorname{Tr} \varrho}\right\} .
\end{aligned}
$$

The term in curly brackets in (3.6) is the analytic continuation of the full interacting Euclidean Green's function $(G(x, y)$ where the point $x$ is taken to lie on a timelike tube at large radius with a small negative imaginary time coordinate and the point $y$ lies on a similar timelike tube with a small positive imaginary time coordinate [18]. The general rule for the expectation value $(\operatorname{Tr} \varrho)^{-1} \operatorname{Tr}\left(\phi\left(x_{1}\right) \phi\left(x_{2}\right) \bar{\phi}\left(x_{3}\right) \ldots \phi\left(x_{n}\right) \varrho\right)$ is that one analytically continues the appropriate $n$-point Green's function from the Euclidean regime to the Lorentzian regime where the points $x_{1}, x_{2}, \ldots x_{n}$ are given small imaginary time coordinates so that

$$
\operatorname{Im}\left(t_{1}\right)<\operatorname{Im}\left(t_{2}\right)<\ldots<\operatorname{Im}\left(t_{n}\right) .
$$

It can be verified that this prescription reproduces the commutation relations (3.4).

The expectation value of the number of outgoing particles between $\omega$ and $\omega+d \omega$ is

$$
\begin{aligned}
\left\langle n_{\text {out }}(\omega)\right\rangle d \omega= & -d \omega \iint f_{\text {out }}(\omega ; x) \overleftrightarrow{\nabla}_{\mu} G(x, y) \\
& \cdot \overleftrightarrow{\nabla}_{v} f_{\text {out }}^{*}(\omega ; y) d \Sigma^{\mu}(x) d \Sigma^{\nu}(y) .
\end{aligned}
$$

This will be infinite because the two surface integrals are over a tube of infinite extent in the time coordinate. However, one can interpret this as a steady finite rate of emission $\dot{n}_{\text {out }}(\omega)$ for an infinite time. To do this replace $f_{\text {out }}^{*}(\omega)$ by $f_{\text {out }}^{*}\left(\omega^{\prime}\right)$ in (3.8). Then

$$
\left\langle n_{\text {out }}\left(\omega, \omega^{\prime}\right)\right\rangle=2 \pi \delta\left(\omega-\omega^{\prime}\right) \dot{n}_{\text {out }}(\omega)
$$

I shall henceforth assume that such a $\delta$ function has been extracted. 

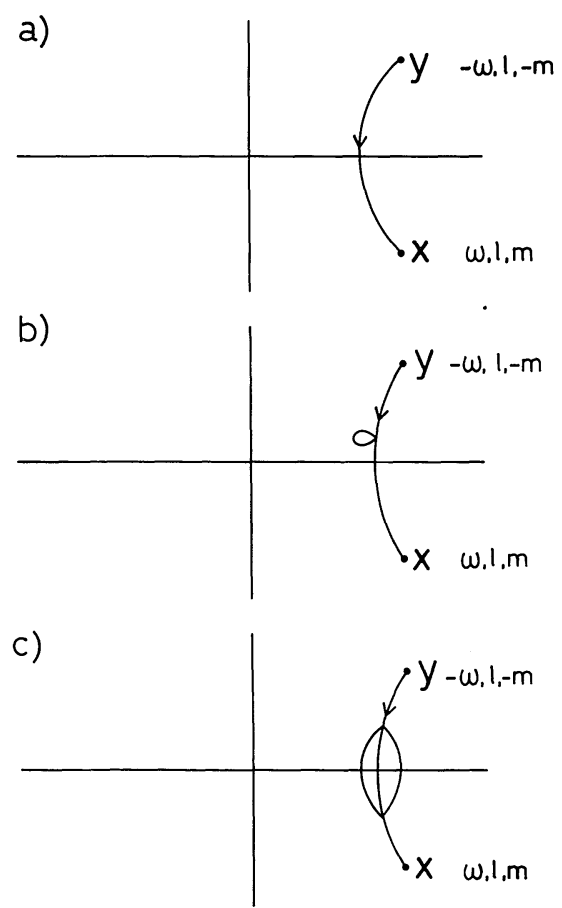

Fig. 3. The lowest order contributions to the number of outgoing particles in thermal equilibrium

One can represent (3.8) as the sum of all diagrams with two external lines going to points $x$ below the real $t$ axis and $y$ above the axis (Fig. 3). I shall adopt the convention that points $x$ and $y$ corresponding to outgoing wave functions are placed on the right of the diagram at large values of $\operatorname{Re}(t)$, though in fact they are integrated over contours just below and just above the real $t$ axis, respectively. The arrows on the lines point from a vertex at which $\phi$ acts to one at which $\bar{\phi}$ acts.

Figure 3 a represents the lowest order diagram in which the points $x$ and $y$ are joined by the free-field propagation $G_{0}(x, y)$. This diagram would be zero if $G_{0}$ were a causal propagator like the flat space propagator at zero temperature because in that case

$$
\psi(z)=\int G_{0}(z, y) \overleftrightarrow{\nabla}_{\mu} f_{\text {out }}^{*}(\omega ; y) d \Sigma^{\mu}(y)
$$

would be zero if the imaginary part of the time coordinate of $z$ were less than that of $y$.

However, by (2.10)

$$
\psi(z)=\left(e^{\beta \omega}-1\right)^{-1} e^{i \omega t} \frac{Y_{\ell m}^{*}(\theta, \phi)}{(4 \pi)^{1 / 2} r} \overleftarrow{R}_{+}^{*}(r)
$$

for $\operatorname{Im}\left(t_{z}\right)<\operatorname{Im}\left(t_{y}\right)$. If one takes the scalar product of this with $f(\omega ; x)$ to give (3.8), one obtains a contribution to $\dot{n}_{\text {out }}(\omega)$ of $(2 \pi)^{-1}\left(e^{\beta \omega}-1\right)^{-1}$. This is zero in the limit of large $\beta$, i.e. low temperature. 

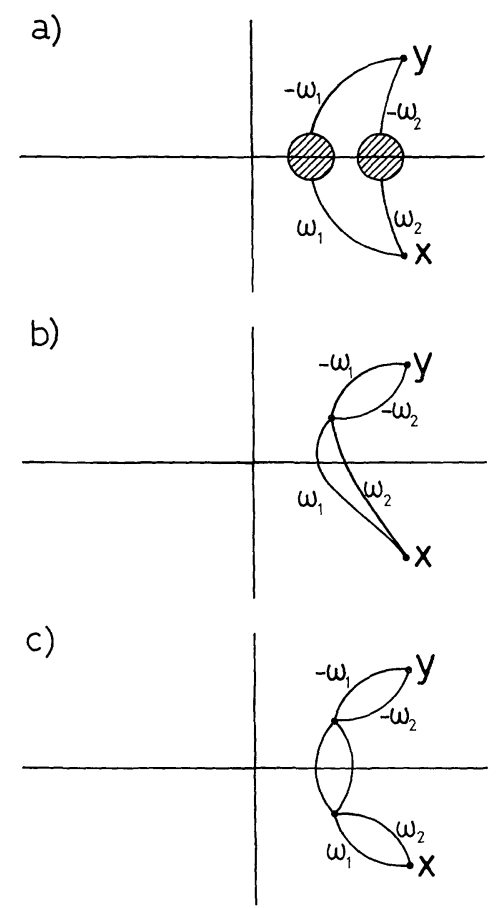

Fig. 4. Diagrams for higher moments of the density matrix

Figure $3 b$ represents the first-order correction to the free-field emission rate. In flat space this would be absorbed into the renormalisation of the mass. However, the finite temperature and curvature of the black hole background will cause the single closed loop with one vertex to differ from its flat space value [12]. Thus Fig. $3 b$ will give a non-zero correction to the rate of emission which can be represented by giving the field an $r$-dependent mass. Similarly, Fig. 3c represents the one-particle irreducible correction at the second order, and so on. One can regard this diagram as corresponding to three particles of the thermal radiation (represented by the three lines crossing the real $t$-axis) which combine to give a single particle which is observed at infinity.

One can also calculate corrections between the numbers emitted in different modes. For example

$$
\left\langle n_{\text {out }}\left(\omega_{1}\right) n_{\text {out }}\left(\omega_{2}\right)\right\rangle=(\operatorname{Tr} \varrho)^{-1}\left(\operatorname{Tr} a_{\text {out }}^{+}\left(\omega_{1}\right) a_{\text {out }}^{+}\left(\omega_{2}\right) a\left(\omega_{1}\right) a\left(\omega_{2}\right) \varrho\right) .
$$

This is shown diagrammatically in Fig. 4. Note that because the operators $a_{\text {out }}\left(\omega_{1}\right)$ and $a_{\text {out }}\left(\omega_{2}\right)$ commute, the surfaces over which the wave functions $f_{\text {out }}\left(\omega_{1}\right)$ and $f_{\text {out }}\left(\omega_{2}\right)$ are integrated can be taken to have the same small negative imaginary part of the time coordinate. This is indicated in Fig. 4 by taking the external lines marked $\omega_{1}$ and $\omega_{2}$ to the same point. Similarly, all the external lines carrying negative frequencies are taken to a point above the real $t$ axis.

In Fig. $4 \mathrm{a}$ the blobs indicate all the diagrams in Fig. 3. These contribute $\left\langle n_{\text {out }}\left(\omega_{1}\right)\right\rangle\left\langle n_{\text {out }}\left(\omega_{2}\right)\right\rangle$, i.e. they give no correlations. However, there are also 


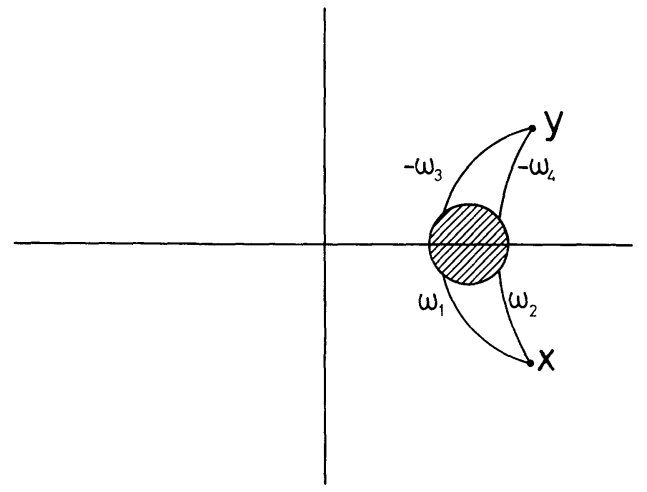

Fig. 5. The diagram for an off-diagonal moment of the density matrix

diagrams like Figs. $4 \mathrm{~b}$ and $4 \mathrm{c}$ in which the $\omega_{1}$ and $\omega_{2}$ lines are connected. These will give correlations between the numbers of particles at different frequencies or angular quantum numbers. These correlations arise because the free-field particle states are not eigenfunctions of the Hamiltonian. One can also measure offdiagonal moments of the density matrix. For example, Fig. 5 represents

$$
(\operatorname{Tr} \varrho)^{-1}\left(\operatorname{Tr} a_{\text {out }}^{x}\left(\omega_{1}\right) a_{\text {out }}^{x}\left(\omega_{2}\right) a_{\text {out }}\left(\omega_{3}\right) a_{\text {out }}\left(\omega_{4}\right) \varrho\right)
$$

where $\omega_{1}, \omega_{2}, \omega_{3}, \omega_{4},>m$ are all different. This will contain a factor of $\delta\left(\omega_{1}+\omega_{2}-\omega_{3}-\omega_{4}\right)$ because of the time invariance of the background metric. In a similar manner one can determine higher moments of the density matrix of the outgoing radiation.

\section{Substracting the Incoming Radiation}

In the previous section it was shown how to use the Euclidean Green's functions to determine the outgoing radiation far from the black hole in the situation that the black hole was in equilibrium with thermal radiation. Although the consideration of such a situation is instructive as a gedanken experiment [6], it is not likely to occur in practice because one cannot make a box which is impervious to gravitons, let alone black holes. What one wants is to calculate the emission from a black hole radiating into empty space. This can be done by adding to the diagrams of Sect. 3a number of extra diagrams with both ingoing and outgoing external lines which have the effect of subtracting out the contributions of the ingoing radiation.

One starts by defining the "Unruh" free-field Green's function $G_{u}(x, y)$ which differs from the Euclidean or "Hartle-Hawking" Green's functions by having no negative frequency component of ingoing radiation.

$$
\begin{aligned}
G_{u}(2,1)= & G_{0}(2,1) \\
& -\frac{1}{4 \pi r^{2}} \sum_{\ell, m} 2 \operatorname{Re} \int_{m}^{\infty} d \omega e^{-i \omega\left(t_{2}-t_{1}\right)}\left(e^{\beta \omega}-1\right)^{-1} \\
& \cdot Y_{\ell m}^{*}\left(\theta_{1}, \phi_{1}\right) Y_{\ell m}\left(\theta_{2}, \phi_{2}\right) \overleftarrow{R}_{-}^{*}\left(r_{1}\right) \overleftarrow{R}_{-}\left(r_{2}\right) .
\end{aligned}
$$


One can represent $G_{u}$ in terms of the Euclidean Green's function $G_{0}$ as shown in Fig. 6. The first diagram on the right corresponds to the $G_{0}(2,1)$ term in $(4.1)$, while the other two diagrams in which the points 1 and 2 are joined to external wave functions from the points $v$ and $u$ correspond to the second term in (4.1). The external lines at $v$ and $u$ are integrated with the wave functions $f_{\text {in }}(\omega, \ell, m)$ and $f_{\text {in }}^{*}(\omega, \ell, m)$ respectively, and the resultant expression is multiplied by $\left(e^{\beta \omega}-1\right)$ and integrated from $\omega=m$ to $\infty$ and summed over $\ell$ and $m$. The point $v$ is integrated over a timelike tube at large radius with a small negative imaginary part to the time coordinate while $u$ has a small positive imaginary part. The points $v$ and $u$ are placed on the left of the diagram at large negative values of real $t$ to indicate that they correspond to incoming wave functions.

The idea is now that to calculate the rate of emission or other moments of the density matrix of outgoing radiation from a black hole in empty space with no incoming radiation, one calculates the same diagrams as in Sect. 3, but with the Euclidean Green's function $G_{0}$ replaced everywhere by $G_{u}$. Using the relation expressed in Fig. 6, one can then reexpress these diagrams in terms of diagrams involving $G_{0}$, but with incoming as well as outgoing external lines. For example, the free-field contribution to the rate of emission given by diagram 3 a becomes the diagram shown in Fig. 7. The first term is what one has in thermal equilibrium and gives a contribution of $(2 \pi)^{-1} \times\left(e^{\beta \omega}-1\right)^{-1}$. The second diagram gives a contribution of $-|A(\omega)|^{2} \cdot(2 \pi)^{-1}\left(e^{\beta \omega}-1\right)^{-1}$ where the factor $|A(\omega)|^{2}$ arises because of the propagation from ingoing modes or outgoing modes. The third diagram gives zero because the lines join positive frequency to positive frequency and negative frequency to negative frequency. The net emission rate is therefore

$$
\begin{aligned}
& \left(1-|A(\omega)|^{2}\right)(2 \pi)^{-1}\left(e^{\beta \omega}-1\right)^{-1} \\
& =|B(\omega)|^{2}(2 \pi)^{-1}\left(e^{\beta \omega}-1\right)^{-1}
\end{aligned}
$$

This is the usual result for the emission rate for a non-interacting field [1].

The first-order correction to the rate of emission in thermal equilibrium is given by Fig. $3 b$ which can be regarded as an $r$-dependent mass correction. After subtracting out the effect of the incoming radiation, one obtains eight diagrams. One can represent all these simply as the $r$-dependent mass correction given by Fig. 8.

The second-order correction to the rate of emission in thermal equilibrium is given by Fig. 3c. This cannot be represented as a local mass correction. After substracting out the incoming radiation, one obtains 84 diagrams. Similarly, one can subtract out the effect of incoming radiation from the diagrams for higher moments of the density matrix of outgoing radiation though the number of diagrams becomes very large at higher orders of perturbation theory.

The procedure for fermions is similar, except that the thermal factor $\left(e^{\beta \omega}-1\right)^{-1}$ is replaced by the Fermi-Dirac factor $\left(e^{\beta \omega}+1\right)^{-1}$. One can also treat gauge fields and their associated Faddev-Popov ghosts in a similar manner to scalar particles. One has to include diagrams with incoming external ghost lines in order to subtract out the incoming ghost-particle radiation.

The next three sections describe applications of this formalism. 

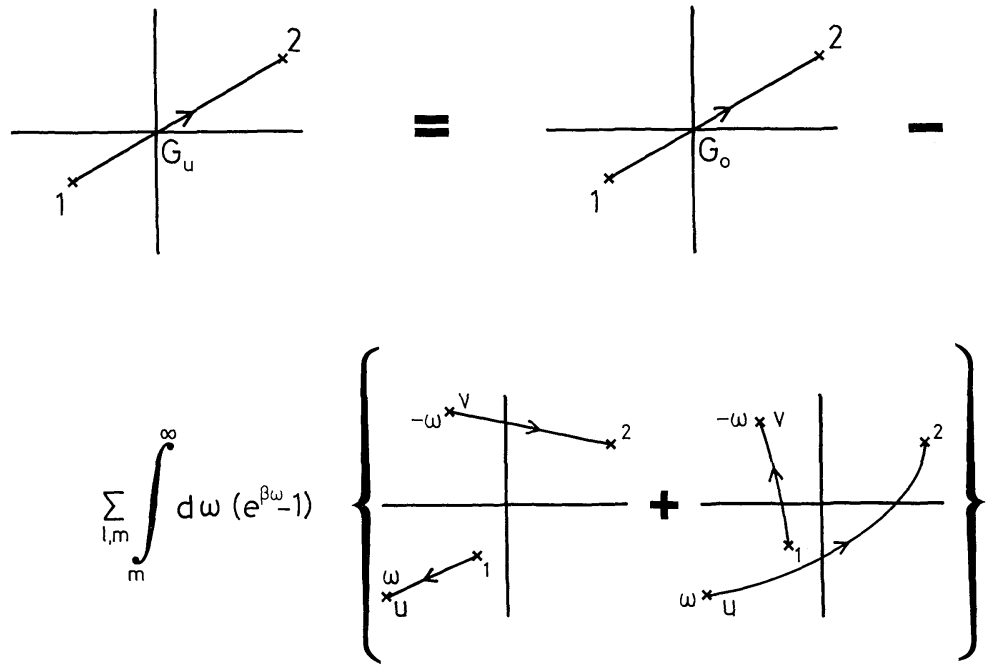

Fig. 6. The Unruh propagator $G_{u}$ expressed in terms of the Euclidean propagator $G_{0}$ plus external lines which subtract out the incoming radiation
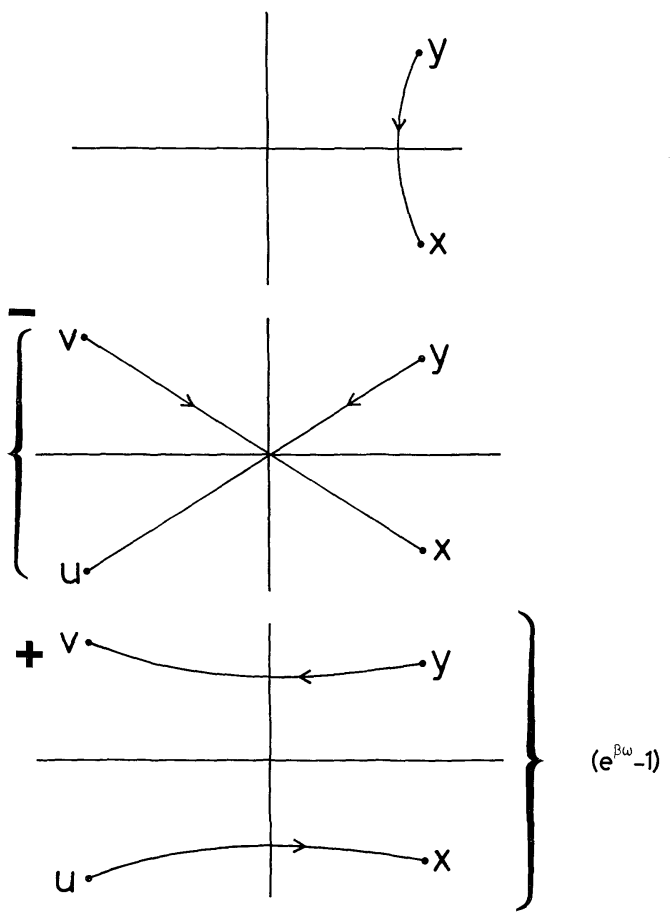

Fig. 7. The free field diagrams for the outgoing radiation from a black hole radiating into empty space 

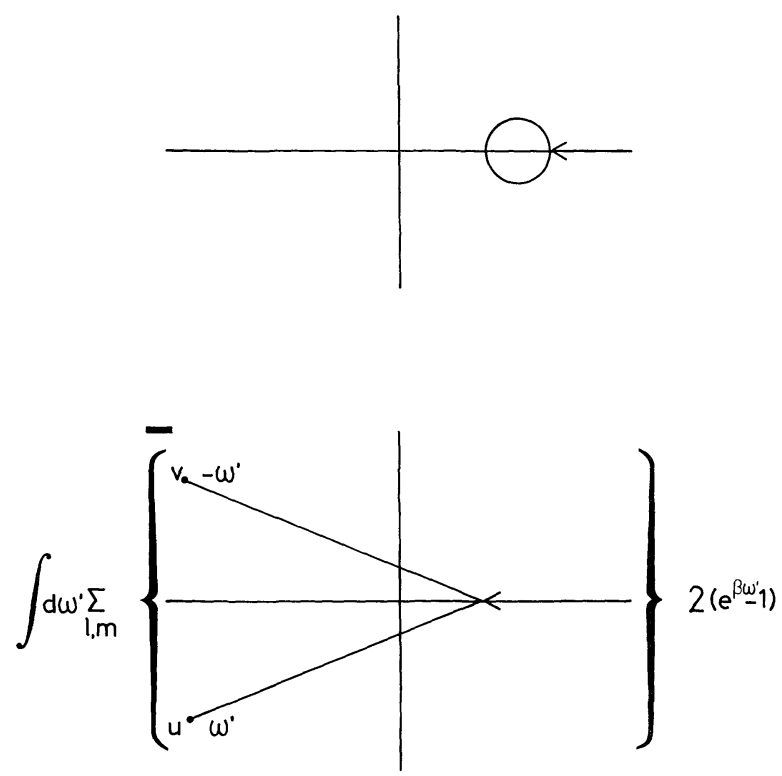

Fig. 8. The position dependent mass correction for a black hole radiating into empty space

\section{Energy-Momentum Tensor etc.}

The partition function $Z$ in thermal equilibrium is given by the trace of the density matrix $\varrho=e^{-\beta H} \cdot \log (Z)$ can be represented as the sum of all closed-loop diagrams with no external lines. In flat space this sum is renormalized to zero, but the periodicity in imaginary time and the curvature will make it non-zero in the Schwarzschild background. By functionally differentiating $\log (Z)$ with respect to the mass parameter which is allowed to be position-dependent, one obtains the expectation value of $|\phi|^{2}$ in thermal equilibrium

$$
\frac{\delta \log (Z)}{\delta m^{2}(x)}=\left\langle|\phi(x)|^{2}\right\rangle
$$

This can be represented as the sum of all diagrams obtained by inserting a vertex on one of the lines of the closed-loop graphs. Candelas [21] has shown that $\left\langle|\phi|^{2}\right\rangle=\frac{2}{3} T^{2}\left(T=(8 \pi M)^{-1}\right)$ at $r=2 M$ for a massless non-interacting complex scalar field in thermal equilibrium. Using a numerical zeta-function technique, Fawcett [12] has shown that $\left\langle|\phi|^{2}\right\rangle$ decreases rapidly at larger radii to the value $\frac{1}{6} T^{2}$ that it would have in flat space at temperature $T$.

It is not easy to substract out the contribution of the incoming radiation from the trace of the density matrix itself. However, it is straightforward to substract it form variational derivatives like (5.1) for $\left\langle|\phi|^{2}\right\rangle$. In the case of a non-interacting field the answer is given in Fig. 8. The first diagram represents the value in thermal 
equilibrium, while the correction terms can be calculated numerically by solving the wave equation [22]. One would expect the resultant $\left\langle|\phi|^{2}\right\rangle$ to be of order $\frac{1}{3} T^{2}$ on the horizon and to decrease to nearly zero at large radius.

The energy-momentum tensor in thermal equilibrium is obtained by functionally differentiating the partition function with respect to the background metric

$$
\left\langle T_{\mu \nu}\right\rangle=2 g^{-1 / 2} \frac{\delta \log (Z)}{\delta g^{\mu \nu}} .
$$

Again this is given by the collection of all closed graphs with a single vertex which now involves derivatives of the Green's function. The lowest order, noninteracting term can be evaluated numerically [12]. One can then substract out the effect of the incoming radiation as for $\left\langle|\phi|^{2}\right\rangle$. This will give an energymomentum tensor which will be regular on the future horizon, but will have a negative energy influx into the hole.

\section{Symmetry Restoration}

It is by now well known that spontaneously broken symmetry is restored at sufficiently high temperatures (see [23] for a review). One might, therefore, expect that it would be restored locally near a sufficiently hot black hole. To investigate this $I$ shall consider first the simplest model of a real scalar field with a negative $(\text { mass })^{2}$ and a quartic interaction:

$$
\mathscr{L}=-\frac{1}{2} \varphi\left(\square+\mu^{2}\right) \varphi+\frac{\lambda}{4} \varphi^{4} .
$$

In flat space at zero temperature the field will have the expection value:

$$
\sigma=\langle\varphi\rangle=\left(\mu^{2} / \lambda\right)^{1 / 2} .
$$

To determine the behaviour at finite temperature in a curved background space, one should consider the effective action $\Gamma$ as a function of the expectation value $\sigma$ of the field. One expresses $\varphi$ as a sum of its expectation value and a fluctuating component $\varrho$ :

$$
\varphi=\sigma+\varrho .
$$

Then

$$
\mathscr{L}=\mathscr{L}_{0}+\mathscr{L}_{1}+\mathscr{L}_{2}+\mathscr{L}_{3},
$$

where

$$
\begin{gathered}
\mathscr{L}_{0}=-\frac{1}{2} \sigma \square \sigma-\frac{1}{2} \mu^{2} \sigma^{2}+\frac{1}{4} \lambda \sigma^{4} \\
\mathscr{L}_{1}=-\sigma \square \varrho-\mu^{2} \sigma \varrho+\lambda \sigma^{3} \varrho \\
\mathscr{L}_{2}=-\frac{1}{2} \varrho \square \varrho-\frac{1}{2} \mu^{2} \varrho^{2}+\frac{3}{2} \lambda \sigma^{2} \varrho^{2} \\
\mathscr{L}_{3}=\lambda \sigma \varrho^{3}+\frac{1}{4} \lambda \varrho^{4} .
\end{gathered}
$$


The effective action $\Gamma(\sigma)$ in the thermal equilibrium is given to one loop order by dropping $L_{1}$ and $L_{3}$ (the term linear, cubic and quartic in $\varrho$ ) and integrating over all fluctuations $\varrho$ which are regular on the Euclidean section and which die off at infinity:

$$
-\Gamma(\sigma)=-\int \mathscr{L}_{0} \sqrt{g} d^{4} x+\log \left\{\int d \varrho \exp \left[-\int \mathscr{L}_{2} \sqrt{g} d^{4} x\right]\right\} .
$$

The expectation value $\sigma$ of the field is determined by the requirement that $\Gamma$ should be a minimum.

$$
\frac{\delta \Gamma}{\delta \sigma}=0=-\square \sigma+\sigma\left(\lambda \sigma^{2}-\mu^{2}+3 \lambda\left\langle\varrho^{2}\right\rangle\right) .
$$

where $\varrho$ behaves like a free field with a position-dependent effective mass

$$
m_{\varrho}^{2}=3 \lambda \sigma^{2}-\mu^{2} \text {. }
$$

When $T \ll \mu$, the expectation value of $\varrho^{2}$ will be very small and the only solution of (6.9) will be $\sigma=\mu \lambda^{-1 / 2}$. When $T>\mu$, the expectation value of $\varrho^{2}$ will be approximately that for a massless scalar field. At large radius $\left\langle\varrho^{2}\right\rangle \sim \frac{1}{12} T^{2}$. So $\sigma=\sigma_{0}$ where

$$
\begin{array}{ll}
\sigma_{0}^{2}=\mu^{2} / \lambda-\frac{1}{4} T^{2}, & T<T_{c} \\
\sigma_{0}=0, & T>T_{c},
\end{array}
$$

where the critical temperature

$$
T_{c}=2 \mu \lambda^{-1 / 2}
$$

Near $r=2 M,\left\langle\varrho^{2}\right\rangle>\frac{1}{12} T^{2}$ and $0<\sigma<\sigma_{0}$.

It is difficult to substract out the effect of the incoming radiation to determine $\Gamma$ for the case of a black hole radiating into empty space. It is however, straightforward to substract out the contribution of the incoming radiation from $\left\langle\varrho^{2}\right\rangle$ in the Eq. (6.9) for $\sigma$. Again one expects $\left\langle\varrho^{2}\right\rangle$ to be small unless $T>\mu$, in which case it can be approximated by the value for a massless field. Equation (6.9) for $\sigma$ will then have a unique solution on the Euclidean section which tends to the zero-temperature value $\mu \lambda^{-1 / 2}$ at large radii. $\sigma$ will decrease at radii less than about $4 \mathrm{M}$ but it will remain non-zero even at $r=2 \mathrm{M}$.

The signal for a phase transition in this situation is not that $\sigma$ vanish (it cannot vanish near the black hole if it is non-zero far away) but that the second functional derivative of the effective action $\frac{\delta^{2} \Gamma}{\delta \sigma(x) \delta \sigma(y)}$ should have a zero mode. In other words, there should be a small perturbation $\delta \sigma$ of the solution of Eq. (6.9) which vanishes at large radii

$$
-\square \delta \sigma+\delta \sigma\left(3 \lambda \sigma^{2}-\mu^{2}+3 \lambda\left\langle\varrho^{2}\right\rangle\right)+3 \lambda \sigma \delta\left(\left\langle\varrho^{2}\right\rangle\right)=0,
$$

where $\delta\left(\left\langle\varrho^{2}\right\rangle\right)$ is the change in $\left\langle\varrho^{2}\right\rangle$ produced by the change in $m_{\varrho}^{2}$ caused by $\delta \sigma$. It is not easy to see whether (6.13) admits a non-trivial solution, but one would not expect it to unless $\sigma$ had been reduced to a value small compared to its zerotemperature value in a region bigger than the typical wavelength $\mu^{-1}$. Now one 
would not expect $\left\langle Q^{2}\right\rangle$ to be significant expect for $r<4 M$ and then only if $T>\mu$ which implies $M<\frac{\mu^{-1}}{8 \pi}$. The expectation value $\sigma$ would then be reduced for $r<4 M$. The region on the Euclidean section on which $r<4 M$ has a proper size of about $9 M$. Thus it seems that $\sigma$ would not be reduced on a large enough region to cause a phase transition, although obviously it is a rather close thing and more careful calculations should be made. One should also work out the physically more relevant case where the scalar field is coupled to a gauge field. In this situation the fluctuations of the gauge field will also help to reduce the expectation value of the field and so restore symmetry.

If there were a phase transition near the black hole associated with the Weinberg-Salam unification at a temperature of about $100 \mathrm{Gev},\left(M=5 \times 10^{10} \mathrm{~g}\right)$, this might produce a sudden burst of emission which might be detectable as a pulse of gamma rays or radio waves. Searches have been made for gamma-ray pulses using the atmosphere as a Cerenkov detector [24]. The results were negative, but the sensitivity was rather low and the upper limit on the frequency of bursts of the order of $10^{32}$ ergs was quite a lot higher than one would expect from the upper limit on the density of primordial black holes given by the gamma-ray background [25]. Radio pulses [26] are potentially a more sensitive method of detecting bursts of emission, but they depend critically on the nature of the burst. Searches for radio pulses have not revealed any evidence for bursts [27].

\section{Particle Antiparticle Asymmetry}

In order to generate an asymmetry between the numbers of particles and antiparticles (or, more precisely, between the occupation numbers of states which are $\mathrm{CP}$ conjugates of each other), three conditions must be satisfied:

1. There must be nonconservation of particle number.

2. There must be $\mathrm{CP}$ violating terms in the lagrangian.

3. The situation must not be invariant under time reversal.

Grand Unified Theories provide examples where the baryon number is not locally conserved although in some cases baryon number minus lepton number is conserved. These theories can also contain CP violating terms. Finally, the early universe provides a background in which there is a well defined arrow of time. It is therefore possible that the observed baryon asymmetry of the universe was generated by the asymmetric decay of superheavy particles when the temperature of the universe was about the Grand Unification Energy $10^{14} \mathrm{Gev}$. (see [28] for a review).

Black holes provide another possible setting for the generation of particleantiparticle asymmetry. In the presence of a black hole the total observed particle number need not be conserved because particles or antiparticles can cross the horizon even if the particle number is locally conserved. A black hole in equilibrium with thermal radiation is invariant under time reversal (or TP reversal in the case of a rotating hole). This means that the numbers of outgoing particles and antiparticles must be equal in such a situation even if there are $\mathrm{CP}$ violating interactions between the quantum fields and the background metric [14]. For 
suppose that the number of outgoing particles was greater than the number of outgoing antiparticles. Then, by CPT, the number of ingoing particles would be less than the number of ingoing antiparticles. Thus one would build up an excess of particles, which cannot happen in thermal equilibrium because there is no arrow of time.

On the other hand, a black hole radiating into empty space does have a well defined arrow of time. In the case of quantum fields that do not interact with each other but only with the background metric, subtracting out the effects of the incoming radiation from the thermal equilibrium results will leave the numbers of outgoing particles and antiparticles equal to each other [14]. However this need not be the case for interacting quantum fields.

A simple model which has non-trivial $C$ and $T$ violations but which has a locally conserved particle number current is provided by three complex scalar fields with quadratic and quartic interactions.

$$
\begin{aligned}
L= & -\sum \bar{\phi}_{i} \square \phi_{i}+\sum m_{i}^{2} \bar{\phi}_{i} \phi_{i}+\sum \lambda_{i} \bar{\phi}_{i}^{2} \phi_{i}^{2} \\
& +g_{1} \bar{\phi}_{2} \phi_{2} \bar{\phi}_{3} \phi_{3}+g_{2} \bar{\phi}_{3} \phi_{3} \bar{\phi}_{1} \phi_{1}+g_{3} \bar{\phi}_{1} \phi_{1} \bar{\phi}_{2} \phi_{2} \\
& +h_{1} \bar{\phi}_{2}^{2} \phi_{3}^{2}+h_{1}^{*} \phi_{2}^{2} \bar{\phi}_{3}^{2}+h_{2} \bar{\phi}_{3}^{2} \phi_{1}^{2}+h_{2}^{*} \phi_{3}^{2} \bar{\phi}_{1}^{2} \\
& +h_{3} \bar{\phi}_{1}^{2} \phi_{2}^{2}+h_{3}^{*} \phi_{1}^{2} \bar{\phi}_{2}^{2} .
\end{aligned}
$$

The $m_{i}$ and $\lambda_{i}$ and $g_{i}$ terms are required for renormalizability. However they are real and do not contribute to $C$ or $T$ violation. The $h_{i}$ terms make the Lagrangian not invariant under the charge conjugation operation which replaces $\phi_{i}$ by $\bar{\phi}_{i}$. Moreover provided that the product $h_{1} h_{2} h_{3}$ is complex, there is no field redefinition which makes the Lagrangian invariant under charge conjugation. Because it is invariant under parity reversal, it must therefore be non-invariant under time reversal. The Lagrangian (7.1) has a global $U(1)$ invariance:

$$
\phi_{i} \rightarrow \phi_{i} e^{i \alpha}, \quad \bar{\phi}_{i} \rightarrow \bar{\phi}_{i} e^{-i \alpha} .
$$

Thus there is a Noether current

$$
J_{\mu}=\frac{1}{i} \sum \bar{\phi}_{i} \stackrel{\partial}{\partial}_{\mu} \phi_{i}
$$

This is locally conserved in the background metric, i.e.

$$
J_{i \mu}^{\mu}=0 \text {. }
$$

To obtain asymmetric emission it is necessary to consider diagrams which involve all three $h_{i}$ vertices. The simplest one-particle irreduccible diagram for the number of outgoing particles of species 1 which does this in thermal equilibrium is shown in Fig. 9. This diagram itself will not give any asymmetry because it corresponds to thermal equilibrium, but asymmetry can arise when the incoming radiation is subtracted out to give diagrams such as the one in Fig. 10. The value of this diagram will be $h_{1} h_{2} h_{3}$ times some number $F$, which may be complex because the diagram is not asymmetric about the real $t$-axis. There will also be a contribution from the complex conjugate diagram, which will be $h_{1}^{*} h_{2}^{*} h_{3}^{*} F^{*}$. On the other hand there will be similar diagrams which give a contribution of $\left(h_{1}^{*} h_{2}^{*} h_{3}^{*} F+h_{1} h_{2} h_{3} F^{*}\right)$ 


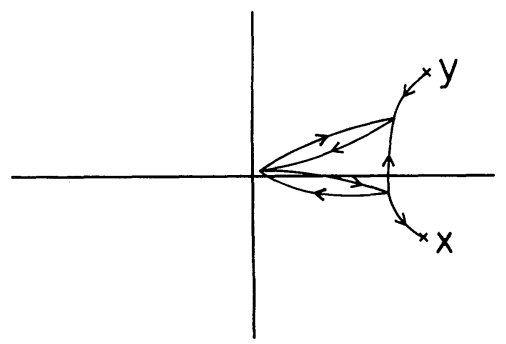

Fig. 9. The simplest one particle irreductible diagram which involves all three $h_{i}$ vertices

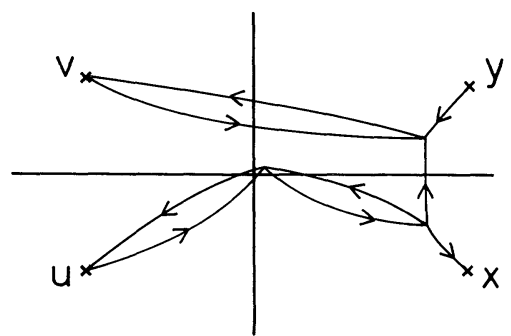

Fig. 10. One of the diagrams that subtract out the incoming radiation from Fig. 9 and can give rise to particle-antiparticle asymmetry

to the number of outgoing antiparticles of species 1 . Thus if $h_{1} h_{2} h_{3}$ is complex, the numbers of outgoing particles and antiparticles of species 1 may differ and this difference can remain when one sums over the other two species as well because the quantities $F$ that appear for the different species will not be related in any simple way. Thus the black hole can emit unequal numbers of particles and antiparticles even though the particle number current is locally conserved: the net flux of particles at infinity is balanced by an equal flux of antiparticles into the black hole.

It is probable that the asymmetric emission of quarks or leptons by black holes significantly bigger than the Planck mass is not of much practical significance because there are tight upper limits on the numbers of such primordial black holes [29]. Rather the importance of this result lies in the fact that it demonstrates that gravitational interactions can generate particle asymmetry even in the presence of a global $U(1)$ symmetry. This is rather analogous to the way in which instantons can violate the conservation law that follows from the $U(1)$ symmetry of chiral invariance [30]. In the context of the early universe it means that CP violating effects near the initial singularity could lead to baryon asymmetry without any need to appeal to Grand Unified Theories.

\section{References}

1. Hawking, S.W.: Commun. Math. Phys. 43, 199-230 (1975)

2. Bardeen, J.M., Carter, B., Hawking, S.W.: Commun. Math. Phys. 31, 181 (1973)

3. Wald, R.M. : Commun. Math. Phys. 45, 9-34 (1975)

4. Parker, L. : Phys. Rev. D12, 1519-25 (1976) 
5. Hawking, S.W.: Phys. Rev. D14, 2460-2473 (1976)

6. Hawking, S.W.: Phys. Rev. D13, 191-7 (1976)

7. Hartle, J.B., Hawking, S.W.: Phys. Rev. D13, 2188-2203 (1976)

8. Gibbons, G.W., Perry, J.M.: Phys. Rev. Lett. 36, 985-987 (1976)

9. Gibbons, G.W., Perry, M.J.: Proc. R. Soc. London A358, 467-94 (1978)

10. Gibbons, G.W., Hawking, S.W.: Phys. Rev. D 15, 2752-6 (1977)

11. Birrel, N.D.: Interacting quantum field theory in curved spacetime. (To appear in Quantum Gravity: A Second Oxford Symposium. Oxford University Press: Oxford, 1981

12. Fawcett, M.: (in preparation)

13. Page, D.N.: Phys. Rev. D 14, 1509-1510 (1976)

14. Toussaint, D., Treiman, S.B., Wilczek, F., Zee, A.: Phys. Rev. D 19, 1036-1050 (1979)

15. Deutsch, D., Candelas, P.: Phys. Rev. D20, 3063-3080, (1979)

16. Kennedy, G.: J. Phys. A11, L173-178 (1978)

17. Kennedy, G., Critchley, R., Dowker, J.S. : Ann. Phys. 125, 346-400 (1980)

18. Hawking, S.W.: Acausal Propagation in Quantum Gravity (To appear in Quantum Gravity: A Second Oxford Symposium, Oxford University Press: Oxford, 1981

19. Unruh, W.G.: Phys. Rev. D14, 870-892 (1976)

20. Christensen, S.M., Fulling, S.A.: Phys. Rev. D 15, 2088-2104 (1977)

21. Candelas, P.: Phys. Rev. D21, 2185-2202 (1980)

22. Whiting, B.: (1981) (in preparation)

23. Linde, A.D.: Rep. Prog. Phys. 42, 389-437 (1979)

24. Porter, N.AA., Weekes, T.C.: MNRAS 183, 205-210 (1978)

25. Page, D.N., Hawking, S.W.: Astrophys. J. 206, 1-7 (1976)

26. Rees, M.J.: Nature 266, 333-335 (1977)

27. Meikle, W.P.F.: Nature 269, 41-43 (1977)

28. Ellis, J., Gaillard, M.K., Nanopoulos, D.V.: Grand Unification and Cosmology. CERN Preprint Th. 2858-CERN

29. Novikov, D., Polnarev, A.G., Starobinsky, A.A., Zeldovich, Ya.B.: Astron. Astrophys. 80, 104-109 (1979)

30. 't Hooft, G.: Phys. Rev. Lett. 37, 8-11 (1976)

Communicated by A. Jaffe

Received February 23, 1981 